ipating individuals may end up visibly nominating papers for mainly political purposes. Worse still, it is not unknown for rival research groups to veto each others' results in order to gain the advantage of publishing first. But this is hardly a problem if both groups have already submitted their respective papers to the preprint archives; they are there for posterity (with their original submission dates), and it is the democratic opinion of the physics community, expressed through the front-end systems, which will ultimately decide on their relative impact.

\section{Some Reservations}

All physicists do not see this kind of approach as the way forward. David Fairlie, a theoretician at the University of Durham, remains unconvinced that frontend systems will have an impact. Says Fairlie: "The idea of nominations is a waste of time; indeed, when all physics articles [on the Web] carry abstracts there should be no need for a review at all! Increasingly, I believe people are finding and learning new ideas through conference participation, rather from the Internet, because they are a captive audience for the duration of a presentation."

Of course, there is little doubt that conventional peer review serves an important function in raising standards by improving papers through requested revisions. This process will still be required, though it seems possible that authors may arrange for it informally. It is certainly in their professional interests to have this kind of check before submitting to the preprint servers, where work becomes instantly accessible worldwide. Voluntary scrutiny already takes place with experimental papers produced by the large international collaborations. Here, an internal committee checks and double-checks the data, analysis, conclusions, and presentation before releasing the work. The result is that experimental papers rarely require any kind of revision before acceptance in the established journals. With more emphasis being placed on preprints as the "finally accepted form" of an author's work, theorists may well follow the trend and "self-police" their own papers. If this were to happen, standards could actually rise of their own accord. Far from leading to an uncontrolled explosion in mediocre preprints, the new developments might lead to an increase in quality, provided there is a widespreadendorsement of the new culture.

How this new culture takes off depends to a strong extent on existing tra- ditions within different scientific disciplines. Outsiders are very often surprised to learn that publishing traditions may vary greatly, even between closely related fields. Bertrand Duplantier, a theoretical physicist at CE Saclay in France collaborates with biologists on topics relating to DNA macromolecule statistics. As an active editor, Duplantier is intrigued by the differences in publishing culture between physicists and biologists. "Preprint distribution is already widely accepted in the high-energy physics community, and the new electronic systems have become very popular. But in biology there is a greater fear of plagiarism at the pre-publication stage, so preprint distribution is relatively less important."

From this it would seem that electronic publishing developments may take place at a different pace within even closely related scientific fields. But one thing that will promote new systems across the board will be rapid advances in communications technology. Ever more sophisticated World-Wide Web browsers are being released, and the ability to interpret and format data in a wide variety of input formats will help smooth over cultural differences. Besides handling HTML (the standard language for presenting material on the Web), browsers are now being developed that can process and display

\section{Soundings}

\section{No Growth for Physical Review}

In order to assure the long-term financial health of its major journals at a time when there is concern about declining subscriptions, the impact of electronic publications, growth in non-US submissions, and declining public and government support for science, the American Physical Society (APS) decided at its Council meeting last November to take measures to ensure that increases in library subscriptions to Physical Review remain at less than $10 \%$ p.a. for the next five years. Aside from the obvious cost reductions, there will be "no overall journal growth" by increasing acceptance standards at a time when the total number of submissions to say Phys Rev A-E and Letters increased by $9 \%$ between 1993 and 1994, and by $5 \%$ between 1994 and 1995 (submissions from eastern Europe increased by $63 \%$ and $52 \%$ in the same periods). Peter Adams, the Phys Rev B editor, writes in Notes to Authors that decisions on publications must now normally be reached after two referring rounds so that extended anonymous review cannot be used to transform an acceptable manuscript into a publishable one. The editors of Phys Rev E wish to publish papers that are not "merely new or free from error" but contain "substantial advancement of previously established knowledge". It is hoped that zero
$\mathrm{T}_{\mathrm{E}} \mathrm{X}$ and $\mathrm{LAT}_{\mathrm{E}} \mathrm{X}$ (the preferred typesetting language of mathematicians and particle physicists) as well as files from popular word-processing packages (which are often used to prepare less mathematical texts). These more flexible browsers will make the construction of on-line journals much simpler, with text conversion of articles to HTML becoming unnecessary. The age of the "do it yourself" journal really does seem to have arrived.

From all this, it can be seen that, whether the momentum for change is driven by technology, or by a real need for peer review reform, real opportunities to change things for the better clearly do exist. For this reason, it is important that the physicists give new systems their full and dedicated support: much will ultimately depend on the dedication and determination of individual practitioners. Only time will tell if those (Ginsparg included) who believe that the advantages make radical change inevitable are correct. However, one could well imagine that, without the active participation of senior figures in the new front-end systems, important new initiatives could wither and die. This would be a great pity for the physics community (which afterall invented WWW technology), and for science publishing in general.

overall growth can be achieved without a "page budget" for editors, but as higher standards increase submissions, this policy may have to be reviewed.

The APS has also decided to try to maintain a long-term policy for page charges that is the same for all fields following recent "experiments" that generated considerably instability. These mostly concerned the elimination of page charges for all submissions to Phys Rev D.The measure had the desired result of more submissions, but cost increases combined with the loss of page-charge income meant that $D$ ran at a loss, even following large increases to the subscription price; libraries which tend to buy packages of journals reacted negatively. The plan is now to raise page charges slightly on all non-compuscript submissions to all journals, and to have by 1999 zero page charges for compuscripts submitted to all Phys Rev journals (only Phys Rev $C$ and $D$ now have zero page charges for compuscripts).

Compuscripts will be redefined to include non$T_{E} X$ desktop wordprocessing formats as well files that are not used in the production process.

Historically, page charges were intended to cover editorial costs, and subscriptions the production and distribution costs, an approach (but not a policy) that was motivated by the desire not to become totally dependent on subscription income. The distinction between these activities has become rather fuzzy in an 
era when the APS processes compuscript submissions, expects to soon have a preprint server (so that authors can benefit from nonanonymous comments before more traditional anonymous refereeing) and is "positioning" to deliver all its research journals online (PRL has been online since July 1995 - with a WWW interface since August 1995 - and the routine conversion of $100 \%$ of MS to electronic formats is in sight).

\section{Hypertext Potential to be Exploited}

Billed as a "fully electronic", peer-reviewed journal in spite of the availability of the traditional paper format, Elsevier Science (http://www.elsevier.nl/) is to launch in early1996 New Astronomy covering all fields of astronomy and astrophysics. It will be made available via WWW and over local area networks (LAN). It is claimed that the WWW edition will represent a new format for publishing research and letter articles through the use of hypertext links to bibliographic databases, scientific data sets, tabular materials, and sets of (colour) figures, animation or sound (which can be part of a submitted article or located elsewhere on WWW). Other features include a short publication time, at least for publication on WWW (one month for the refereeing process; one month between acceptance and WWW release) and free subscriptions (covering the WWW version together with the LAN and printed editions) during the start-up period of at least 1996.

\section{Program Library Integrated}

The Computer Physics Communications Internal Program Library which holds some 1500 programs, with about 150 new programs added annually, (http://www.cs.qub.ac.uk/cpc/; cpc@qub.ac.uk) was established in 1969 at the Queen's University of Belfast under a UK government grant as a self-supporting repository for the storage and dissemination of refereed computer programs for non-profit use, descriptions of which were published in Computer Physics Communications (CPC). As from January 1996, the Library has become an integral part of the journal so that a subscription to CPC includes access to the library (users in subscribing institutions can register directly).

\section{IOPP Journals Go Online}

The IOPP (http://www.iop.org/) now has 14 journals on WWW and the remaining 17 will follow within a few weeks. Any researcher in a subscribing institution, once registered, will be able to access via WWW papers up to three weeks before paper publication (initially as HTML files for headers and interface, Adobe Acrobat PDF and PostScript files for full text, maths and graphics, and $\mathrm{T}_{\mathrm{E}} \mathrm{X}$ for some journals).

Physics Online Library Launched Springer has set up the Physics Online Library $(P O L)$ - a digital collection of original peer-reviewed contributions coming from a variety of sources, as well as electronic articles in all areas of physics. $P O L$ will incorporate online versions of Zeitschrift für Physik A, B, C, and $D$ to be released in the course of 1996 .

This comes at a time when Springer has announced that a Helpdesk on Internet will be

\section{Archiving On-line Journals}

A number of purely electronic (without printed versions), peer-reviewed journals in the physical sciences or related areas are now being offered, generally free of charge to anyone with WWW access. Claims include: extremely rapid publication; instant world-wide distribution: increased readership (since often no subscription fees); immediate downloading and printing of articles; a level of service far beyond the capabilities of paper journals (video and audio capabilities; viewing of digitized items, e.g., full-colour photographs); rapid communicate with authors through direct email links within the text; the posting of comments; debate and discussion groups moderated by an expert; thematic searching. However, most journals do not discuss archiving: some claim "universal access to their results in perpetuity" but do not explain how this is done. Only occasionally is a specific archiving procedure mentioned, as detailed in the table below giving: Title. Start of publication/structure/fees/access/publisher Archiving procedure

Complexity International April 1994/continuous addition/no fees/WWW/Australian National Univ. http://life.anu.edu.au/ci/ci.html

Electronic J. of Combinatorics Nov. 1994/3 issues/no fees/WWW/Georgia Tech in cooperation with the American Mathematical Soc. - http://ejc.math.gatech.edu:8080/Journal/journalhome.html

Internet J. of Nitride Semiconductor Research Jan. 1996/no fees for readers; page charges/WWW/under the auspices of the Materials Research Soc. - http://nsr.mij.mrs.org/

J. of Molecular Modelling March 1995/continuous addition/fees for institutions \& individuals/FTP ; WWW abs. of accepted papers/Univ. Erlangen-Erlangen in cooperation with Springer - http://derioc1.organik.uni-erlangen.de/ info/JMOLMOD/jmolinfo.html

2 year min. FTP availablity; CD-ROM issued

J. of Universal Computer Science Nov. 1994/12 issues/none in '95-96/WWW; some features only with Hyper-G viewers/Univ. of Graz in Collab. with Springer - http://hgiicm.tu-graz.ac.at/CJUCS_root

CD-ROM issued

Mathematical Physics Electronic Journal (March 1995/1 issue/no fees/ WWW; PostScript files, wih logo, volume, paper number, \& time stamps/University of Texas - http://www.ma.utexas.edu/mpej/MPEJ.html

Files maintained by Univ. of Texas; printed copy of each volume sent to US Library of Congress

Molecular Vision Oct. 1995/1 issue/no fees/WWW/Emory Univ., USA http://www.cc.emory.edu/MOLECULAR_VISION?index.html

Theory \& Applications of Categories July-1995/continuous addition/individuals free; institutional subscription/WWW/ Int. Steering Committee- $h$ ttp://www.tac.mta.ca/tac/ Electronic archives maintained by

available for subscribers to the 110-year old Landolt-Bernstein data collection, together with electronic tools and complete electronic versions to improve retrieval and document delivery procedures.

\section{Few-Body Systems Electronic}

The electronic version of Springer's FewBody Systems (http://fbs.kfunigraz.ac.at/Cfbs/) has finished a one-year test phase. A subscription (either individual with password protection or institutional with machine protection) to the printed plus electronic versions is now needed to access all its features. The clients Harmony (for UNIX) and Amadeus (for MS Windows) are needed to fully exploit the Graz University Hyper-G hypermedia system which the journal uses. Access via World-Wide Web or Gopher is possible, but some advanced features of Hyper-G (e.g., following links in PostScript documents) are not supported.

\section{Will SGML Make a Comeback ?}

Everyone who publishes WWW pages must have a working knowledge of the Hyper-Text Markup Language (HTML) which allows text to embed simple formatting information and links. But HTML is a moving target: HTML 2.0 is an Internet Draft and many issues (tables, math, character sets, etc.) do not form part of it, and HTML 3.0 is in flux. HTML is one instance of the infinite family of Standardised Generalised Markup Language (SGML) metalanguages used for defining markup. SGML is useful for archiving text in databases and for reproduction on different media. With the notable exception of the American Physical Society, publishers have tended to adopt in-house versions of SGML.

The widespread use of HTML demonstrates that SGML could be useful for networked information. One route is to treat SGML like a specialized data format, and to launch specialized or general-purpose SGML browsers to display data. The other (better) route is to integrate SGML awareness beyond HTML into browsers. Either way, display of an SGML document could be controlled by a style sheet to define new logical tags and their default visual interpretation, using a small number of display primitives ("bold", "line break", etc.) to specify the rendition of each element type. For "wellknown" Document Type Definitions (DTDs) like HTML, style sheets could be distributed with the browser, or built in. For other DTDs, the browser could fetch a style sheet from the server. A common (or meta) style-sheet language for browsing and forms, at least, must be agreed on to take serious advantage of SGML. The Document Style Semantics and Specification Language (DSSSL) has been proposed as a general set of transformation rules, with DSSSL Lite and FOSI being used for document display. A simpler approach would to reach agreement on a minimal SGML document that is sent by a server. The browser can then do a minimal amount of parsing provided a few additional rules are agreed on. A more radical approach is to use programming languages such as Java which allow relatively difficult to develop applications, downloaded while browsing, to format text and formulae. 